\title{
Testing a double AGN hypothesis for Mrk 273
}

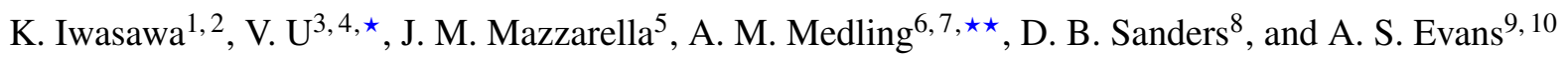 \\ ${ }^{1}$ Institut de Ciències del Cosmos (ICCUB), Universitat de Barcelona (IEEC-UB), Martí i Franquès, 1, 08028 Barcelona, Spain \\ e-mail: kazushi.iwasawa@icc.ub.edu \\ 2 ICREA, Pg. Lluís Companys 23, 08010 Barcelona, Spain \\ 3 Department of Physics and Astronomy, 900 University Avenue, University of California, Riverside, CA 92521, USA \\ ${ }^{4}$ Department of Physics and Astronomy, 4129 Frederick Reines Hall, University of California, Irvine, CA 92697, USA \\ 5 IPAC, MS 100-22, California Institute of Technology, Pasadena, CA 91125, USA \\ 6 Cahill Center for Astronomy and Astrophysics, California Institute of Technology, MS 249-17, Pasadena, CA 91125, USA \\ 7 Research School of Astronomy \& Astrophysics, Australian National University, Canberra, ACT 2611, Australia \\ 8 Institute for Astronomy, University of Hawaii, 2680 Woodlawn Drive, Honolulu, HI 96822, USA \\ 9 Department of Astronomy, University of Virginia, Charlottesville, VA 22903-2325, USA \\ 10 National Radio Astronomy Observatory, 520 Edgemont Road, Charlottesville, VA 22903-2475, USA
}

Received 27 July 2017 / Accepted 4 November 2017

\section{ABSTRACT}

\begin{abstract}
The ultra-luminous infrared galaxy (ULIRG) Mrk 273 contains two infrared nuclei, N and SW, separated by 1 arcsecond. A Chandra observation has identified the SW nucleus as an absorbed X-ray source with $N_{\mathrm{H}} \sim 4 \times 10^{23} \mathrm{~cm}^{-2}$ but also hinted at the possible presence of a Compton-thick AGN in the N nucleus, where a black hole of $\sim 10^{9} M_{\odot}$ is inferred from the ionized gas kinematics. The intrinsic X-ray spectral slope recently measured by NuSTAR is unusually hard $(\Gamma \sim 1.3)$ for a Seyfert nucleus, for which we seek an alternative explanation. We hypothesize a strongly absorbed X-ray source in $\mathrm{N}$, of which X-ray emission rises steeply above $10 \mathrm{keV}$, in addition to the known X-ray source in SW, and test it against the NuSTAR data, assuming the standard spectral slope $(\Gamma=1.9)$. This double $\mathrm{X}$-ray source model gives a good explanation of the hard continuum spectrum, deep Fe $\mathrm{K}$ absorption edge, and strong Fe $\mathrm{K}$ line observed in this ULIRG, without invoking the unusual spectral slope required for a single source interpretation. The putative X-ray source in $\mathrm{N}$ is found to be absorbed by $N_{\mathrm{H}}=1.4_{-0.4}^{+0.7} \times 10^{24} \mathrm{~cm}^{-2}$. The estimated 2-10 keV luminosity of the $\mathrm{N}$ source is $1.3 \times 10^{43} \mathrm{erg} \mathrm{s}^{-1}$, about a factor of 2 larger than that of SW during the NuSTAR observation. Uncorrelated variability above and below $10 \mathrm{keV}$ between the Suzaku and NuSTAR observations appears to support the double source interpretation. Variability in spectral hardness and $\mathrm{Fe} \mathrm{K}$ line flux between the previous $\mathrm{X}$-ray observations is also consistent with this picture.
\end{abstract}

Key words. galaxies: nuclei $-\mathrm{X}$-rays: galaxies

\section{Introduction}

Major mergers of gas-rich galaxies appear to play an important role in the creation of ultra-luminous infrared galaxies (ULIRGs; Sanders \& Mirabel 1996). Dense molecular gas channelled into the nuclear region in the process of a merger drives intense star formation (Barnes \& Hernquist 1992). Numerical simulations predict that the nuclear black holes of merging galaxies accrete gas simultaneously (e.g. Hopkins et al. 2005). Those active black holes (active galactic nuclei, hereafter AGN) are naturally obscured heavily during the ULIRG phase. As a result, they are sometimes difficult to identify due to nuclear obscuration and the surrounding star formation activity that masks their AGN signatures.

Mrk 273 is a nearby ULIRG $\left(L_{\mathrm{ir}}=10^{12.21} L_{\odot}\right)$ at $z=0.038$, exhibiting a long tidal tail (Fig. 1), which is a clear signature of a recent galaxy merger. There are three radio components, N, SW, and SE (Condon et al. 1991) in the nuclear region (Fig. 1), two of which ( $\mathrm{N}$ and $\mathrm{SW}$ ) coincide with near-infrared nuclei with a separation of 1 arcsec imaged by HST/NICMOS (Scoville et al. 2000).

\footnotetext{
$\star$ University of California Chancellor's Postdoctoral Fellow.

$\star \star$ Hubble Fellow.
}

The SW component is a point-like, red, near-infrared source and is identified as an absorbed hard X-ray source (Iwasawa et al. 2011) with $N_{\mathrm{H}} \simeq 4 \times 10^{23} \mathrm{~cm}^{-2}$ (Iwasawa 1999; Xia et al. 2002; Ptak et al. 2003; Balestra et al. 2005; Iwasawa et al. 2011). Together with the optical emission-line characteristics (Colina et al. 1999), SW is likely to contain an obscured Seyfert nucleus. However, much of the infrared luminosity of Mrk 273 must originate from the other nucleus N (see Iwasawa et al. 2011, and references therein), where a concentration of a large amount of molecular gas is found (Downes \& Solomon 1998) and intense star formation is taking place (Carilli \& Taylor 2000; Bondi et al. 2005). While the starburst appears to dominate the power output, near-infrared integral-field spectroscopy has revealed a strongly rotating disc in $\mathrm{N}$, from which a black hole of $10^{9} M_{\odot}$ is inferred (U et al. 2013; see also Klöckner \& Baan 2004; Rodríguez Zaurín et al. 2014). Given the environment of the $\mathrm{N}$ nucleus, the black hole is expected to be buried deeply in the dust and gas shrouds.

Although SW dominates the Chandra hard X-ray (3-7 keV) image, the 6-7 keV narrow-band image containing the Fe $\mathrm{K}$ line $(6.4 \mathrm{keV})$ shows a possible extension towards $\mathrm{N}$. This is in contrast to the point-like, continuum-only (4-6 keV) image centred on SW, and points to $\mathrm{N}$ being the origin of the enhanced Fe K line (Iwasawa et al. 2011). The Chandra data quality was 

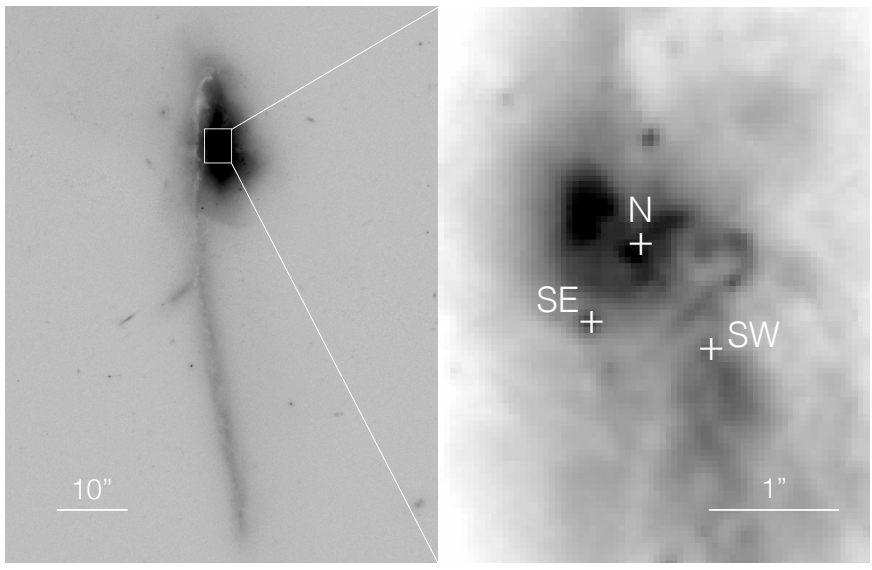

Fig. 1. Left: HST/ACS $I(F 814 W)$ band image of Mrk 273. The image orientation is north up, east to the left. The box indicates the zoomed region in the right panel. Right: the nuclear region of Mrk 273 is shown. Positions of the three radio components, N, SW, and SE (Condon et al. 1991) are denoted by plus symbols. Much of the far-infrared emission arises from the $\mathrm{N}$ nucleus while Chandra imaging found the SW nucleus to be a primary hard X-ray source (see Iwasawa et al. 2011 for $\mathrm{X}$-ray source identification).

insufficient to be conclusive, but if this is true, it suggests the presence of an X-ray source that exhibits a strong Fe $\mathrm{K}$ line and faint underlying continuum in the $\mathrm{N}$ nucleus. These characteristics fit an X-ray spectrum of a Compton-thick AGN (with $N_{\mathrm{H}} \geq 10^{24} \mathrm{~cm}^{-2}$ ), of which hard X-ray emission may rise above $10 \mathrm{keV}$.

The recently published NuSTAR observation of Mrk 273 (Teng et al. 2015) detected hard X-ray emission up to $30 \mathrm{keV}$. Teng et al. estimated the spectral slope of the absorbed continuum of $\Gamma \simeq 1.4$. This slope is corrected for absorption and the effects of Compton scatterings in the obscuring medium. A simple absorbed power-law fit, as usually used in a spectral analysis of an X-ray survey, gives a harder slope of $\Gamma \simeq 1.3$ (Sect. 2). It is rare to find such a hard intrinsic X-ray slope among AGN. The photon index falls just below the entire photon index distribution measured for the Swift/BAT hard X-ray selected AGN and lies outside the $3 \sigma$ region of the intrinsic scatter around the mean photon index $(\Gamma=1.94$ for Type 1 AGN and $\Gamma=1.84$ for Type 2 AGN; Ueda et al. 2014). Theoretically, extreme conditions are required to produce a power-law X-ray slope harder than $\Gamma=1.5$ in the popular disc-corona model (Haardt \& Maraschi 1993). Here, a single absorbed source, the SW nucleus, is assumed to account for the entire NuSTAR spectrum, as the double nucleus cannot be resolved spatially by X-ray telescopes other than Chandra. We suppose that, in addition to the SW source, a Compton-thick AGN is present in N, which elevates the hard X-ray emission above $10 \mathrm{keV}$, offering a natural explanation for the hard NuSTAR spectrum. We explore this possibility of a double X-ray source assuming the standard X-ray spectral parameters usually found for AGN, namely, a photon index $\Gamma=1.9$ and solar metallicity.

The cosmology adopted here is $H_{0}=70 \mathrm{~km} \mathrm{~s}^{-1} \mathrm{Mpc}^{-1}$, $\Omega_{\Lambda}=0.72, \Omega_{\mathrm{M}}=0.28$.

\section{NuSTAR spectrum}

The NUSTAR mission observed Mrk 273 during guaranteed time on 2013 November 14 (ObsID 6000202802). We used cleaned event files processed by nupipeline using the latest calibration files. The net exposure time is $70 \mathrm{ks}$ for both FPMA and
FPMB modules. After checking that the spectra from the two modules agree within errors, we used the averaged spectrum for the spectral analysis below. The data above $4 \mathrm{keV}$ are used to avoid any extranuclear emission in the soft band. We binned the spectrum so that each bin has more than 32 source counts and used the $\chi^{2}$ minimization method to search for best fits. The errors quoted in each parameter are of $1 \sigma$.

First, the 4-40 keV spectrum is fitted by a power law modified by a cold absorber in the line of sight. Various effects of Compton scattering in the absorbing medium, for example, reflected continuum emission when a torus-type geometry is assumed for an absorber, are ignored here. This is to give an idea of the spectral shape and to make a direct comparison of the result with the general properties of AGN possible, as X-ray survey data are usually analysed in this way. A narrow Gaussian is added to describe the $6.4 \mathrm{keV} \mathrm{Fe} \mathrm{K}$ line. Figure 2a shows the NUSTAR data and the deviation from the best-fitting absorbed power-law model, which finds $\Gamma=1.26 \pm 0.10$ and $N_{\mathrm{H}}=(3.0 \pm 0.6) \times 10^{23} \mathrm{~cm}^{-2}$ (as measured in the galaxy rest frame). The remarkably hard spectral slope is the primary characteristic that led us to seek an alternative explanation involving double AGN, as argued in Sect. 1. The Fe K line is found at rest energy of $6.39 \pm 0.06 \mathrm{keV}$ with intensity of $(4.6 \pm 1.0) \times$ $10^{-6} \mathrm{ph} \mathrm{cm}^{-2} \mathrm{~s}^{-1}$. The equivalent width (EW) of the line to the absorbed continuum is $0.36 \pm 0.08 \mathrm{keV}$. We note that this model systematically overestimates the data in the 7-9 $\mathrm{keV}$ range, where the $\mathrm{Fe} \mathrm{K}$ absorption edge (the threshold energy at $7.1 \mathrm{keV}$ for neutral $\mathrm{Fe}$ ) is present. The above fit of the absorbing column is primarily driven by the continuum rollover at the low energy side. The mismatch of the Fe $\mathrm{K}$ absorption band indicates that the Fe absorption edge is deeper than predicted by the best-fit $N_{\mathrm{H}}$. This can be explained by increased Fe metallicity (relative to the solar abundance of Anders \& Grevesse 1989). While other elements are tied together and independent from $\mathrm{Fe}$, when $\mathrm{Fe} \mathrm{K}$ metallicity is fitted $N_{\mathrm{H}}(\mathrm{Fe})$ is found to be $(4.5 \pm 0.9) \times 10^{23} \mathrm{~cm}^{-2}$, while $N_{\mathrm{H}}=(3.2 \pm 0.5) \times 10^{23} \mathrm{~cm}^{-2}$ for the other elements. Although statistical evidence for excess Fe metallicity is not strong, it is suggestive for the proposed twocomponent model as discussed below.

In Sect. 1, we mentioned the possible presence of a Compton-thick AGN in the $\mathrm{N}$ nucleus in addition to the SW nucleus, suggesting that the hard NuSTAR continuum as measured above may result from elevated continuum emission above $10 \mathrm{keV}$ due to a strongly absorbed source in N. Here, a surmised $\mathrm{N}$ source would have $N_{\mathrm{H}} \sim 10^{24} \mathrm{~cm}^{-2}$. The above inspection of the NUSTAR spectrum gives two more properties to favour this hypothesis: the deep Fe $\mathrm{K}$ absorption edge and the large $\mathrm{EW}$ of the $\mathrm{Fe} \mathrm{K}$ line; however, these properties are not as strong as the spectral slope. An additional strongly absorbed source would enhance the total Fe edge depth without invoking the excess Fe metallicity. The observed Fe line EW of $0.36 \mathrm{keV}$ is larger than expected for an absorbing torus with $N_{\mathrm{H}} \sim 3 \times 10^{23} \mathrm{~cm}^{-2}(E W \simeq 0.2 \mathrm{keV}$; Ikeda et al. 2009; Ghisellini et al. 1994; Awaki et al. 1991). In fact, the spectral fit using the MYTORUS model presented in Teng et al. (2015) appears to underestimate the Fe K line flux (their Fig. 5). Extra line emission from $\mathrm{N}$, as hinted by the Chandra observation (see Sect. 1), could account for the excess line emission.

We proceed to model the NUSTAR spectrum with two absorbed nuclei, SW and $\mathrm{N}$. The $\mathrm{N}$ nucleus is hypothesized to have an X-ray source absorbed by a larger $N_{\mathrm{H}}$ than that for SW. We assume that each nucleus has an X-ray source with the standard power-law slope of $\Gamma=1.9$ (e.g. Nandra \& Pounds 1994; Ueda et al. 2014). Effects of reflection within the respective 


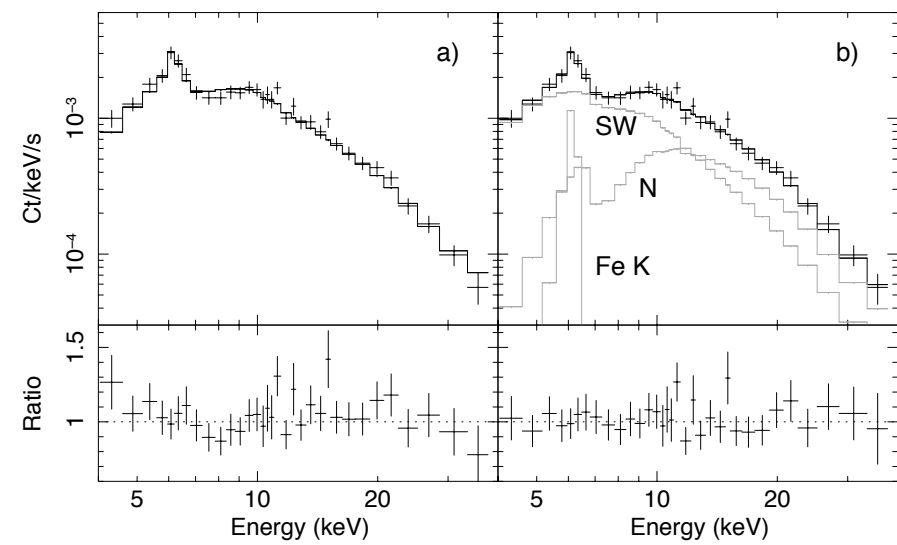

Fig. 2. Panel a: count rate spectrum of Mrk 273 from NuSTAR plotted with the best-fit absorbed power-law model (Model (i) of Table 1 in histogram). The model includes a Gaussian line for the Fe $\mathrm{K}$ line at $6.4 \mathrm{keV}$. The data to model ratio is plotted in the bottom panel. The best-fit slope is very hard $(\Gamma=1.26)$. Panel $b$ : the same data plotted with the two source model, Model (iii). The best-fit models for the two absorbed continuum sources (labelled SW and $\mathrm{N}$ ) and the total Fe K line (labelled $\mathrm{Fe} \mathrm{K}$ ) are drawn in light grey histograms.

absorbers computed by Monte Carlo simulations assuming a torus geometry (e-torus: Ikeda et al. 2007; details of the usage are described in Awaki et al. 2009) are included. Fe K line emission, which is not included in e-torus, is modelled by a Gaussian, which accounts for a sum of the line emission from both nuclei. Since the inclination and opening angles of the tori of the respective nuclei cannot be constrained independently, we made the following assumptions of the torus geometry: both tori are inclined at $55^{\circ}$ (which is the inclination angle measured for the gas disc at $\mathrm{N}$ by $\mathrm{U}$ et al. 2013 but not known for SW); the opening angles are $50^{\circ}$ for $\mathrm{SW}$ and $10^{\circ}$ for $\mathrm{N}$, as a relatively large opening of the torus is expected for SW exhibiting Seyfert 2 characteristics (Colina et al. 1999; U et al. 2013) while a small opening for the dense-gas filled $\mathrm{N}$ nucleus. Solar metallicity is assumed for both absorbers.

This double source model describes the data well (Fig. 2b), and the quality of fit is comparable to or even better than those single source models (Table 1). The model agrees with the Fe K absorption band well. The assumed steep spectral slope fits better the data at the high energy end $(25-40 \mathrm{keV})$, which results in a slightly smaller $\chi^{2}$ than in Model (ii). The absorbing columns are found to be $N_{\mathrm{H}}=2.6_{-1.1}^{+0.9} \times 10^{23} \mathrm{~cm}^{-2}$ for SW and $N_{\mathrm{H}}=1.4_{-0.4}^{+0.7} \times 10^{24} \mathrm{~cm}^{-2}$ for $\mathrm{N}$. The fit suggests that the hypothetical $\mathrm{N}$ nucleus is absorbed by a Thomson depth of unity, in agreement with a suspected Compton-thick AGN. According to the fit, 87 per cent of the observed $4-8 \mathrm{keV}$ flux comes from the SW component while the $\mathrm{N}$ component, which is faint at lower energies, rises sharply towards $10 \mathrm{keV}$ and exceeds SW at $\sim 12 \mathrm{keV}$ upwards (see Fig. 2b). The observed 4-8 keV and 10-30 keV fluxes and the intrinsic continuum source luminosities in the $2-10 \mathrm{keV}$ bands for the SW and N components, estimated from the fit, are given in Table 2. Intrinsically, the hidden $\mathrm{N}$ nucleus appears to be a factor of 2 more luminous than the SW nucleus.

The measured $\mathrm{Fe} \mathrm{K}$ line flux found in the above fit is $(4.1 \pm 1.0) \times 10^{-6} \mathrm{ph} \mathrm{cm}^{-2} \mathrm{~s}^{-1}$; there are contributions from both nuclei. According to Ikeda et al. (2009), $E W=0.18 \mathrm{keV}$ is expected for an illuminated torus with $N_{\mathrm{H}}=2.6 \times 10^{23} \mathrm{~cm}^{-2}$, as obtained for SW. The corresponding line intensity is $2.2 \times$ $10^{-6} \mathrm{ph} \mathrm{cm}^{-2} \mathrm{~s}^{-1}$. The rest of the line flux $((1.9 \pm 1.0) \times$
Table 1. Spectral fits to the NUSTAR data.

\begin{tabular}{cccc}
\hline \hline \multicolumn{5}{c}{ (i) Absorbed power law } \\
$\Gamma$ & $N_{\mathrm{H}}$ & $\chi^{2} /$ d.o.f. \\
$1.26 \pm 0.10$ & $3.0 \pm 0.6$ & - & $56.70 / 55$ \\
\hline \multicolumn{4}{c}{ (ii) With enhanced Fe metallicity } \\
$\Gamma$ & $N_{\mathrm{H}}$ & $N_{\mathrm{H}}(\mathrm{Fe})$ & $\chi^{2} /$ d.o.f. \\
$1.41 \pm 0.11$ & $3.2 \pm 0.5$ & $4.5 \pm 0.9$ & $48.80 / 54$ \\
\hline \multicolumn{5}{c}{ (iii) $\mathrm{SW} \mathrm{\&} \mathrm{N} \mathrm{components}$} \\
$\Gamma$ & $N_{\mathrm{H}}^{\mathrm{SW}}$ & $N_{\mathrm{H}}^{\mathrm{N}}$ & $\chi^{2} /$ d.o.f. \\
1.9 & $2.6_{-1.1}^{+0.9}$ & $14_{-4}^{+7}$ & $46.20 / 54$ \\
\hline
\end{tabular}

Notes. Model (i) is a single power law modified by cold absorption. The value $\Gamma$ is the photon index and $N_{\mathrm{H}}$ is the hydrogen equivalent column density in units of $10^{23} \mathrm{~cm}^{-2}$, measured in the galaxy rest frame. Model (ii) is the same as Model (i), except that $N_{\mathrm{H}}$ for Fe is fitted independently from the other elements. Model (iii) assumes both SW and $\mathrm{N}$ sources have the standard power-law slope $\Gamma=1.9$ and are modified by varying absorbing columns. Unlike in Models (i) and (ii), the effects of Compton scattering within the respective absorbing tori, computed by e-torus (Ikeda et al. 2009), are included (see text for details). A narrow Gaussian for the $\mathrm{Fe} \mathrm{K}$ line at $6.4 \mathrm{keV}$ is included in all the fits. The fits with Models (i) and (iii) correspond to Figs. 2a and b, respectively.

Table 2. Decomposed fluxes and luminosities.

\begin{tabular}{ccccc}
\hline \hline Nucleus & $F_{4-8}$ & $F_{10-30}$ & $L_{2-10}$ & $L_{10-30}$ \\
\hline $\mathrm{SW}$ & 3.6 & 12 & 6.2 & 4.8 \\
$\mathrm{~N}$ & 5.6 & 18 & 13 & 10 \\
\hline
\end{tabular}

Notes. Estimated fluxes in the $4-8 \mathrm{keV}$ and $10-30 \mathrm{keV}$ bands in units of $10^{-13} \mathrm{erg} \mathrm{s}^{-1} \mathrm{~cm}^{-2}$, and the intrinsic luminosities in unit of $10^{42} \mathrm{erg} \mathrm{s}^{-1}$, corrected for absorption and reflection for the SW and $\mathrm{N}$ nuclei, based on the two-component decomposition (Model (iii) in Table 1 and Fig. 2b). The Fe $\mathrm{K}$ emission-line flux is excluded from the measured fluxes.

$10^{-6} \mathrm{ph} \mathrm{cm}^{-2} \mathrm{~s}^{-1}$ ) can then be attributed to $\mathrm{N}$. The corresponding $\mathrm{EW}$ with respect to the $\mathrm{N}$ continuum is $E W \simeq 0.7 \mathrm{keV}$, which is expected for $N_{\mathrm{H}} \simeq 1 \times 10^{24} \mathrm{~cm}^{-2}$, in agreement with the estimated $N_{\mathrm{H}}$ for $\mathrm{N}$. The same spectral fit using absorption and reflection spectra with Fe line emission, computed by other codes, for example, MYTORUS (Murphy \& Yaqoob 2009) instead of e-torus gives a consistent result.

The spectrum taken from the region of the $\mathrm{N}$ nucleus in the earlier Chandra observation shows marginal evidence of a strong $6.4 \mathrm{keV}$ line (Iwasawa et al. 2011) with intensity of $1.4 \pm$ $0.7 \mathrm{ph} \mathrm{cm}^{-2} \mathrm{~s}^{-1}$, which is comparable to the above estimate of the Fe line in $\mathrm{N}$ based on the NuSTAR. In addition to emission from a putative AGN, the strong starburst taking place in $\mathrm{N}$ could generate hot gas emitting high-ionization $\mathrm{Fe}$ (mainly Fe xxv) emission, which may contribute to the 6-7 keV image extension towards $\mathrm{N}$ seen by Chandra. There are strong cases for such high-ionization Fe emission in the ULIRG Arp 220 (Iwasawa et al. 2005, 2009; Teng et al. 2009, 2015) and the LIRG NGC 3690E (Ballo et al. 2004; Ptak et al. 2015). Assuming the high-ionization $\mathrm{Fe}$ emission is proportional to star formation rate, we estimated its line flux based on the measurements for Arp 220 and NGC 3690E, taking into account the source distances. This line flux is found to be $(4-8) \times 10^{-7} \mathrm{ph} \mathrm{cm}^{-2} \mathrm{~s}^{-1}$, that is approximately $10-20 \%$ of the total line flux detected with NUSTAR. 


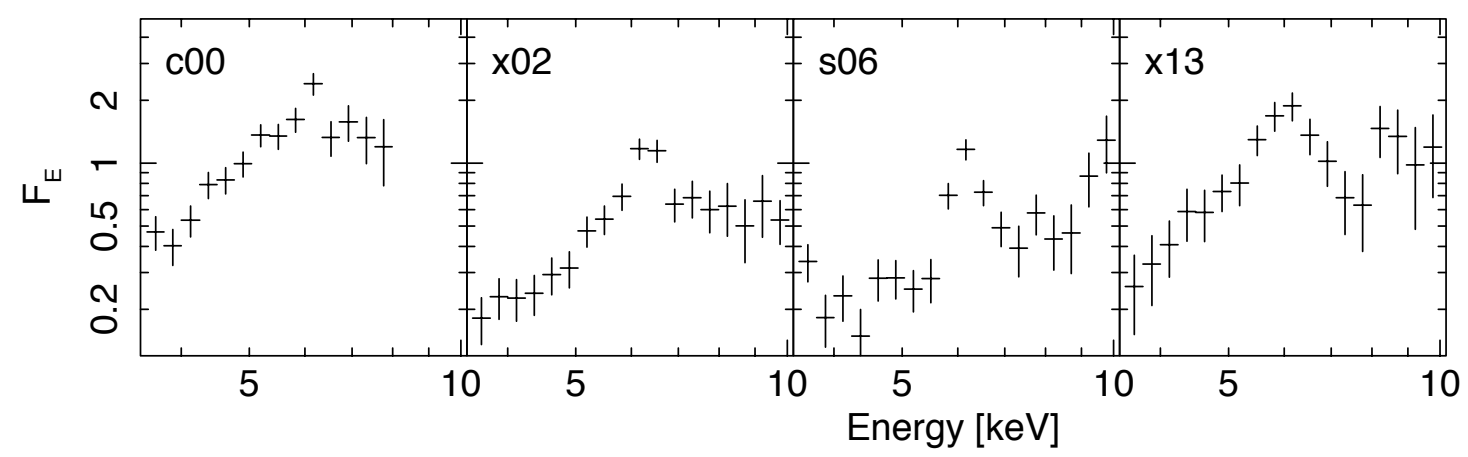

Fig. 3. X-ray spectra of Mrk 273 observed at the four epochs (Table 3). The data are plotted in flux density unit of $10^{-13} \mathrm{erg} \mathrm{s}^{-1} \mathrm{~cm}^{-2} \mathrm{keV}^{-1}$.

Table 3. X-ray observations of Mrk 273.

\begin{tabular}{clcrcccc}
\hline \hline Observation & Observatory & $\begin{array}{c}\text { Date } \\
\text { yyyy-mm-dd }\end{array}$ & ObsID & Detector & $\begin{array}{c}\text { Exposure } \\
\text { ks }\end{array}$ & $\begin{array}{c}\text { Counts } \\
\mathrm{ct} \mathrm{s}^{-1}\end{array}$ & Band \\
\hline c00 & Chandra & $2000-04-19$ & 809 & ACIS-S & 45 & 533 & $4-8$ \\
x02 & XMM-Newton & $2002-05-07$ & 0101640401 & EPIC & $18 / 22$ & 573 & $4-10$ \\
s06 & Suzaku & $2006-07-07$ & 701050010 & XIS/PIN & $80 / 77$ & $626 / 714$ & $4-10 / 15-40$ \\
x13 & XMM-Newton & $2013-11-04$ & 0722610201 & EPIC & $3.5 / 20$ & 478 & $4-10$ \\
n13 & NuSTAR & $2013-11-04$ & 6000202802 & FPMA/B & 70 & 2930 & $4-40$ \\
\hline
\end{tabular}

Notes. XMM-Newton carries three EPIC cameras (pn, MOS1, and MOS2). The exposure times for the pn and two MOS cameras are shown separately: (pn)/(average of MOS1 and MOS2), but the source counts are from all the three cameras. We use Suzaku data from the four XIS cameras and HXD-PIN. The exposure times, source counts, and energy bands in which the source counts were measured are given separately. All the XIS source counts are summed together.

\section{Spectral variability}

There are previous X-ray observations of Mrk 273 mainly at energies lower than $10 \mathrm{keV}$ and the source flux is variable. According to the spectral decomposition, while the observed continuum flux below $10 \mathrm{keV}$ is dominated by $\mathrm{SW}$, the two nuclei may have comparable contributions to the Fe $\mathrm{K}$ line. Spectral variability, including the Fe line, accompanied by total X-ray brightness changes may provide a clue to the reality of two-component modelling. We used four other X-ray observations of Mrk 273 performed with the Chandra X-ray Observatory (Chandra), Suzaku, and XMM-Newton between 2000 and 2013 (Table 3, they are labelled c00, x02, s06, and x13). There are two XMM-Newton observations, one of which (x13) was carried out simultaneously with the NUSTAR observation (n13). The 4-10 keV data of the EPIC pn and two MOS cameras of XMM-Newton and Suzaku XIS and the 4-8 keV data of Chandra were used. Their spectra are shown in Fig. 3. The 4-8 keV X-ray source flux observed in c00 dropped significantly during x02 and s06 and then recovered during X13 and n13.

Among these previous observations, marginal detection in the 15-40 keV band with the Suzaku HXD-PIN during s06 (Teng et al. 2009) may have potential significance to our hypothesis. Our own analysis (with the 2009-Aug. version of hxdpinxbpi and the "tuned" ver. 2.0 background) obtained $15-40 \mathrm{keV}$ flux of $(4.3 \pm 1.1) \times 10^{-12} \mathrm{erg} \mathrm{s}^{-1} \mathrm{~cm}^{-2}$ (the error is statistical uncertainly only) and the spectral data are plotted in Fig. 4, along with the 3-10 keV XIS data taken in the same observation and the NUSTAR data. The source excess $(\sim 3 \%)$ is slightly larger than the systematic uncertainty of the background ( $1.3 \%$ in $15-40 \mathrm{keV}$; Mizuno et al. 2008) and the detection remains marginal. Supposing this PIN measurement is real, Fig. 4 shows that the observed $15-40 \mathrm{keV}$ flux is comparable between $\mathrm{s} 06$ and $\mathrm{n} 13\left(f_{15-40}=(3.3 \pm 0.3) \times 10^{-12} \mathrm{erg} \mathrm{s}^{-1} \mathrm{~cm}^{-2}\right)$, whilst the Suzaku XIS flux below $10 \mathrm{keV}$ is more than a factor

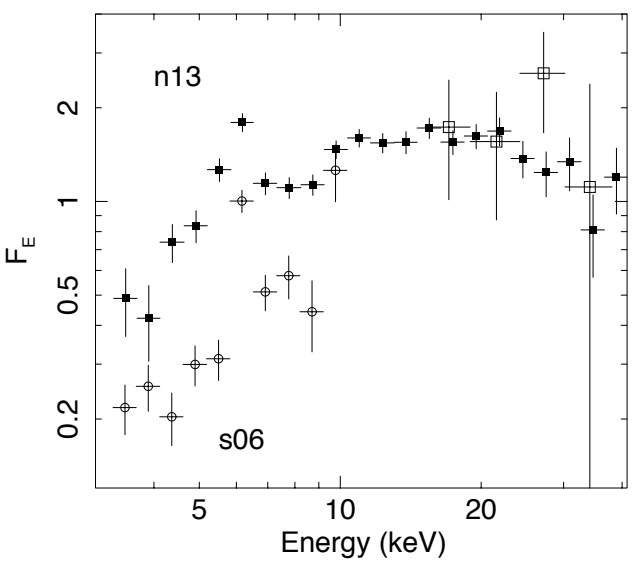

Fig. 4. Same as Fig. 3 but the Suzaku XIS (open circles) and PIN (open squares) data from s06 are plotted with the NUSTAR broadband data (filled squares).

of 2 fainter than that of NuSTAR (see Table 4). This uncorrelated variability above and below $10 \mathrm{keV}$ suggests two distinct sources are present in the respective bands, subject to the reliability of the PIN detection, in agreement with the NuSTAR spectral decomposition (Fig. 2b). In the double source picture, the SW source is variable while the $\mathrm{N}$ source maintains similar brightness. The same spectral model fitted to the NuSTAR data (Model (iii) of Table 1) gives a good description of the Suzaku spectrum by changing only the continuum normalization for SW and the Fe line flux.

Now we look at spectral variability below $10 \mathrm{keV}$ between the four observations via a simple modelling. All the spectra are modelled by an absorbed power law plus a narrow Gaussian for the Fe K line. We assume a power-law slope of $\Gamma=1.26$ obtained from the NUSTAR spectrum (Sect. 2). The column density of cold 
Table 4. Variability between four obserations.

\begin{tabular}{cccc}
\hline \hline $\begin{array}{c}\text { Obs } \\
(1)\end{array}$ & $\begin{array}{c}N_{\mathrm{H}} \\
(2)\end{array}$ & $\begin{array}{c}I_{\mathrm{Fe}} \\
(3)\end{array}$ & $\begin{array}{c}f_{4-8} \\
(4)\end{array}$ \\
\hline $\mathrm{c} 00$ & $2.9 \pm 0.5$ & $3.3 \pm 1.0$ & $5.2 \pm 0.7$ \\
$\mathrm{x} 02$ & $3.8 \pm 0.5$ & $1.7 \pm 0.5$ & $2.0 \pm 0.3$ \\
$\mathrm{~s} 06$ & $3.5 \pm 0.7$ & $2.3 \pm 0.5$ & $1.5 \pm 0.3$ \\
$\mathrm{x} 13$ & $3.1 \pm 0.6$ & $3.6 \pm 1.4$ & $3.8 \pm 0.6$ \\
\hline
\end{tabular}

Notes. (1) Observations indicated in Table 1; (2) absorbing column density $N_{\mathrm{H}}$ measured in the galaxy rest frame in units of $10^{23} \mathrm{~cm}^{-2}$; (3) iron line intensity $I_{\mathrm{Fe}}$ in units of $10^{-6} \mathrm{ph} \mathrm{cm}^{-2} \mathrm{~s}^{-1}$, as observed, without correction for absorption; (4) source flux in the 4-8 keV band in units of $10^{-13} \mathrm{erg} \mathrm{s}^{-1} \mathrm{~cm}^{-2}$. The flux is measured for the continuum (the Fe $\mathrm{K}$ line flux has been subtracted).

absorber $N_{\mathrm{H}}$, power-law normalization and Fe line strength are left variable between the observations. If SW is solely responsible for the observed X-ray emission, the measured $N_{\mathrm{H}}$ is the absorbing column towards the SW nucleus, while in the context of the double source model, it is a measure of spectral hardness in the 4-10 keV band of the summed spectrum of both nuclei. The spectral shown in Fig. 3 have been corrected for the detector response curve (Iwasawa et al. 2012) and when data from multiple detectors are available (XMM-Newton EPIC, and Suzaku XIS), they are averaged for the convenience of a visual comparison. Spectral fitting was performed for uncorrected data from the individual detectors jointly, using the detector responses and background to obtain the spectral parameters of interest. The absorbing column density, Fe line intensity, and 4-8 keV flux estimated from the spectral fits are shown in Table 4.

Among the four observations, the continuum source is bright in $\mathrm{c} 00$ and $\mathrm{x} 13$ and faint in $\mathrm{x} 02$ and s06. The variability results on $N_{\mathrm{H}}$ and Fe line flux between the bright and faint states appear to be more consistent with the " $\mathrm{SW}+\mathrm{N}$ " hypothesis than that of SW alone, as explained below.

The Fe line flux dropped when the $4-8 \mathrm{keV}$ continuum flux was low (x02 and s06) and rose back when the continuum brightened (x13), suggesting that the line responds to the continuum illumination. However, the correlation plot (Fig. 5) shows a positive offset in line flux at zero continuum intensity. As the SW nucleus dominates the observed 4-8 keV continuum, the continuum variability should be driven by SW. The responding Fe line is therefore attributed to that from SW and the offset line flux $\left(\simeq 1.4 \times 10^{-6} \mathrm{ph} \mathrm{cm}^{-2} \mathrm{~s}^{-1}\right)$ can be considered to originate from $\mathrm{N}$. This line flux decomposition roughly agrees with that from the EW argument for the NUSTAR data (Sect. 2). This interpretation relies on the condition that the Fe line emitter in SW lies sufficiently close to the central source so that it follows the continuum variability within the intervals between the observations (a few years, corresponding to a $\sim \mathrm{pc}$ in physical scale).

On the other hand, the inferred $N_{\mathrm{H}}$ is larger when the 4-8 keV flux is low (Table 4). In the dual AGN scenario, this would be a natural consequence. When the $4-8 \mathrm{keV}$ flux, which is largely due to $\mathrm{SW}$, is lowered, a relative contribution of the more strongly absorbed $\mathrm{N}$ became larger because $\mathrm{N}$ appears to remain in similar flux level, as discussed above (see Fig. 4); this results in a harder spectrum that manifests itself as a larger absorbing column as measured. We assume that column densities obscuring the two nuclei remain the same, which may not necessarily hold. The variability of $N_{\mathrm{H}}$ in absorbed AGN is not uncommon (e.g. Risaliti et al. 2002). However, since the absorbing clouds in the line of sight do not know what the continuum

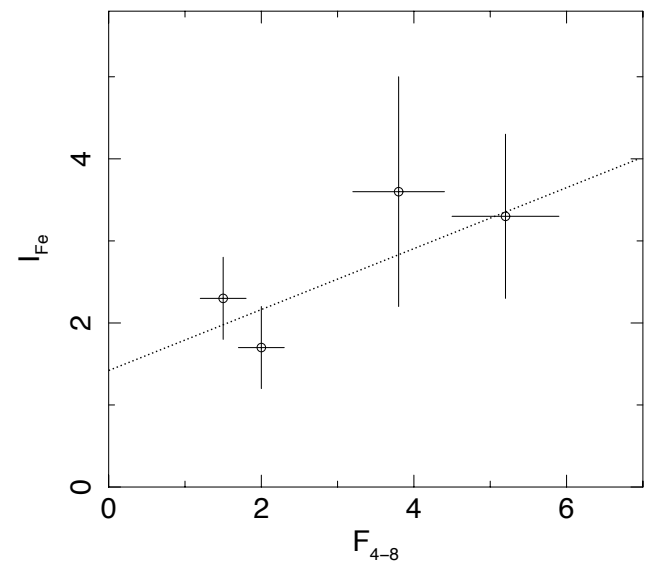

Fig. 5. Correlation diagram of the $\mathrm{Fe} \mathrm{K}$ line and $4-8 \mathrm{keV}$ continuum fluxes between the four observations. The $4-8 \mathrm{keV}$ continuum flux and the Fe line intensity are in units of $10^{-13} \mathrm{erg} \mathrm{s}^{-1} \mathrm{~cm}^{-2}$ and $10^{-6} \mathrm{ph} \mathrm{cm}^{-2} \mathrm{~s}^{-1}$, respectively.

source does, $N_{\mathrm{H}}$ variability should be independent of the continuum flux and any correlated behaviour with continuum brightness as seen here would not exist. A larger number of measurements are needed to test the randomness of $N_{\mathrm{H}}$ variability.

In summary, the disconnected flux variability below and above $10 \mathrm{keV}$ seen between s06 and n13 appears to support two distinct sources, as inferred by the NUSTAR spectral decomposition, indicating that the SW source is significantly variable while the $\mathrm{N}$ source is more stable between the observations. The Fe K line flux and the spectral hardness, as a function of source brightness, also behave as expected from the double source hypothesis, under reasonable conditions.

\section{Discussion}

If the SW nucleus alone accounts for the X-ray emission of Mrk 273 observed with NUSTAR, some extreme conditions are required to produce the unusually hard continuum slope and the excess Fe metallicity. For the former, for instance, a compact corona (thus injection of soft photons from the disc is minimized) with a large optical depth, for example, $\tau \sim 10$, may be a requirement. Blazar-like jet emission, which could produce a hard X-ray spectrum, is unlikely because SW is a weak radio source (Condon et al. 1991). While SW as the sole $\mathrm{X}$-ray source cannot be ruled out, the introduction of an additional, strongly absorbed X-ray source hypothesized in $\mathrm{N}$, offers a more natural explanation for the NUSTAR spectrum with X-ray characteristics that are normal for AGN (Sect. 2) and various spectral variabilities (Sect. 3). U et al. (2013) also argued for a buried AGN in $\mathrm{N}$, using the $\mathrm{H}_{2}$ outflow energetics and the photoionization condition inferred from the $[\mathrm{Si} \mathrm{vI}] / \mathrm{Br} \gamma$ ratio (see also Rodríguez Zaurín et al. 2014) in support of the putative Compton-thick AGN. It might be possible to test the two source hypothesis/scenario with Fe $\mathrm{K}$ narrowband imaging (Iwasawa et al. 2011) of improved data quality (new $200 \mathrm{ks}$ Chandra data have been acquired recently; PI: S. Veilleux), but we probably have to wait for imaging with a future highresolution hard X-ray telescope to be decisive.

The 2-10 keV absorption-corrected luminosity of the hypothetical $\mathrm{N}$ nucleus is estimated to be $1.3 \times 10^{43} \mathrm{erg} \mathrm{s}^{-1}$, comparable to Seyfert nuclei. The bolometric AGN luminosity of $\mathrm{N}$ is then $\sim 2 \times 10^{44} \mathrm{erg} \mathrm{s}^{-1}$ when the bolometric correction of Marconi et al. (2004) is used. It is about two orders of magnitude 
below the total infrared luminosity, indicating that the AGN does not dominate the total energy production in Mrk 273. With a black hole mass of $1 \times 10^{9} M_{\odot}$, the Eddington ratio is about $10^{-3}$. NGC 6240 is a well-known example of a double AGN system in LIRGs with two Compton-thick nuclei (Komossa et al. 2003). If proved true, the double AGN in Mrk 273 would be similar to those found in LIRGs Mrk 266 (Mazzarella et al. 2012) and IRAS 20210+1121 (Piconcelli et al. 2010), in which one of the two AGN in each system is Compton thick.

Acknowledgements. This research made use of data obtained from NuSTAR, Chandra X-ray Observatory, XMM-Newton and Suzaku, reduced and analysed by software provided by HEASARC, the Chandra X-ray Center, and the $X M M-N e w t o n$ Science Operation Centre. K.I. acknowledges support by the Spanish MINECO under grant AYA2016-76012-C3-1-P and MDM-2014-0369 of ICCUB (Unidad de Excelencia "María de Maeztu"). V.U. acknowledges funding support from the University of California Chancellor's Postdoctoral Fellowship. Support for AMM is provided by NASA through Hubble Fellowship grant \#HST-HF2-51377 awarded by the Space Telescope Science Institute, which is operated by the Association of Universities for Research in Astronomy, Inc., for NASA, under contract NAS5-26555.

\section{References}

Anders, E., \& Grevesse, N. 1989, Geochim. Cosmochim. Acta, 53, 197 Awaki, H., Koyama, K., Inoue, H., \& Halpern, J. P. 1991, PASJ, 43, 195 Awaki, H., Terashima, Y., Higaki, Y., \& Fukazawa, Y. 2009, PASJ, 61, S317 Balestra, I., Boller, T., Gallo, L., Lutz, D., \& Hess, S. 2005, A\&A, 442, 469 Ballo, L., Braito, V., Della Ceca, R., et al. 2004, ApJ, 600, 634

Barnes, J. E., \& Hernquist, L. 1992, ARA\&A, 30, 705

Bondi, M., Pérez-Torres, M.-A., Dallacasa, D., \& Muxlow, T. W. B. 2005, MNRAS, 361, 748
Carilli, C. L., \& Taylor, G. B. 2000, ApJ, 532, L95

Colina, L., Arribas, S., \& Borne, K. D. 1999, ApJ, 527, L13

Condon, J. J., Huang, Z.-P., Yin, Q. F., \& Thuan, T. X. 1991, ApJ, 378, 65 Downes, D., \& Solomon, P. M. 1998, ApJ, 507, 615

Ghisellini, G., Haardt, F., \& Matt, G. 1994, MNRAS, 267, 743

Haardt, F., \& Maraschi, L. 1993, ApJ, 413, 507

Hopkins, P. F., Hernquist, L., Cox, T. J., et al. 2005, ApJ, 630, 705

Ikeda, S., Awaki, H., \& Terashima, Y. 2009, ApJ, 692, 608

Iwasawa, K. 1999, MNRAS, 302, 96

Iwasawa, K., Sanders, D. B., Evans, A. S., et al. 2005, MNRAS, 357, 565

Iwasawa, K., Sanders, D. B., Evans, A. S., et al. 2009, ApJ, 695, L103

Iwasawa, K., Mazzarella, J. M., Surace, J. A., et al. 2011, A\&A, 528, A137

Iwasawa, K., Mainieri, V., Brusa, M., et al. 2012, A\&A, 537, A86

Klöckner, H.-R., \& Baan, W. A. 2004, A\&A, 419, 887

Komossa, S., Burwitz, V., Hasinger, G., et al. 2003, ApJ, 582, L15

Marconi, A., Risaliti, G., Gilli, R., et al. 2004, MNRAS, 351, 169

Mazzarella, J. M., Iwasawa, K., Vavilkin, T., et al. 2012, AJ, 144, 125

Mizuno, T., Fukazawa, Y., Takahashi, H., et al. 2008, JX ISAS Suzaku Memo

Murphy, K. D., \& Yaqoob, T. 2009, MNRAS, 397, 1549

Nandra, K., \& Pounds, K. A. 1994, MNRAS, 268, 405

Piconcelli, E., Vignali, C., Bianchi, S., et al. 2010, ApJ, 722, L147

Ptak, A., Heckman, T., Levenson, N. A., Weaver, K., \& Strickland, D. 2003, ApJ, 592, 782

Ptak, A., Hornschemeier, A., Zezas, A., et al. 2015, ApJ, 800, 104

Risaliti, G., Elvis, M., \& Nicastro, F. 2002, ApJ, 571, 234

Rodríguez Zaurín, J., Tadhunter, C. N., Rupke, D. S. N., et al. 2014, A\&A, 571, A57

Sanders, D. B., \& Mirabel, I. F. 1996, ARA\&A, 34, 749

Scoville, N. Z., Evans, A. S., Thompson, R., et al. 2000, AJ, 119, 991

Teng, S. H., Veilleux, S., Anabuki, N., et al. 2009, ApJ, 691, 261

Teng, S. H., Rigby, J. R., Stern, D., et al. 2015, ApJ, 814, 56

U, V., Medling, A., Sanders, D., et al. 2013, ApJ, 775, 115

Ueda, Y., Akiyama, M., Hasinger, G., Miyaji, T., \& Watson, M. G. 2014, ApJ, 786, 104

Xia, X. Y., Xue, S. J., Mao, S., et al. 2002, ApJ, 564, 196 